\title{
Supporting Protocol-Based Care in Medicine via Multiple Coordinated Views
}

\author{
Wolfgang Aigner and Silvia Miksch \\ Institute of Software Technology \& Interactive Systems (ISIS) \\ Vienna University of Technology \\ Favoritenstraße 9-11/188, A-1040 Vienna, Austria, Europe \\ \{aigner, silvia\}@asgaard.tuwien.ac.at
}

\begin{abstract}
Computer supported protocol-based care aims to aid physicians in the treatment process. The main focus of current research is directed towards the formal methods and representations used "behind the scenes" of such systems. This work on the contrary, is situated at the human end of the human-machine chain.

We describe the development of interactive visualization methods to support protocol-based care. We provide multiple simultaneous views to cover different aspects of a complex underlying data structure of treatment plans and patient data. The tightly coupled views use visualization methods well-known to domain experts and are designed to facilitate users' tasks. The views are based on the concepts of clinical algorithm maps and LifeLines which have been extended in order to cope with the powerful and expressive plan representation language Asbru.

The user-centered development approach applied for these interactive visualization methods has been guided by user input gathered via a user study, design reviews, and prototype evaluations.
\end{abstract}

Keywords - Information visualization; multiple views; protocol-based care; treatment plans; user centered design; medical informatics

\section{Introduction}

Visualization tools have been used in the medical domain for a long time. The majority of applications belong to the field of scientific visualization, for example 3D volume visualization tasks, $\mathrm{x}$-ray, or computer tomography visualizations. When it comes to information visualization tasks, the situation is quite different. Abstract data, such as patient data, treatment data, or lab results have mostly been spared out in terms of visualization so far.

Computer support in protocol-based care is a relatively new field of medical informatics. Its core entity, medical treatment plans, are complex documents, currently mostly in the form of prose text including tables and figures [10]. Protocol-based care utilizes clinical protocols to assist in quality improvement and reduce process irregularities. Such clinical protocols are a standard set of tasks that define precisely, how different classes of patients should be managed or treated. They can be seen as reusable definitions of a particular care process. Treatment planning covers the whole process of selecting and executing a particular clinical protocol for a specific patient. Several research projects are dealing with the formalization of this kind of documents in order to facilitate computer based execution support (see [26] for an overview). Hereby, knowledge acquisition, formalizing unstructured treatment documents, creating domain models, data abstraction, executing plans (semi-) automatically, and the like are the major concerns of research. Not much work has been done in order to communicate the computerized treatment plans to the medical staff and even less for combining this with the presentation of patient data when treating a patient along a plan. Currently, most of the data is organized in paper based records including general patient data, treatment steps, lab results, medications, and much more, making it hard to get a comprehensive overview or relate data of different kinds to each other. The integrated visualization of medical treatment plans and patient data could be of great assistance to ease the complex and demanding tasks physicians have to face daily.

\subsection{The Plan Representation Language Asbru}

Asbru is a time-oriented, intention-based, skeletal planspecification representation language that is used in the $A s$ gaard Project $^{1}$ to represent clinical guidelines and protocols in XML. Asbru can be used to express clinical protocols as skeletal plans [12] that can be instantiated for every patient.

1 In Norse mythology, Asgaard was the home of the gods. It was located in the heavens and was accessible only over the rainbow bridge, called Asbru (or Bifrost) (For more information about the Asgaard project see http://www. asgaard.tuwien.ac.at). 
It was designed specific for the set of plan-management tasks [21]. Asbru enables the designer to represent both the prescribed actions of a skeletal plan and the knowledge roles required by the various problem-solving methods for performing the intertwined supporting subtasks. The major features of Asbru are that;

- prescribed actions and states can be continuous;

- intentions, conditions, and world states are temporal patterns;

- uncertainty in both temporal scopes and parameters can be flexibly expressed by bounding intervals;

- plans might be executed in sequence, all or some plans in parallel, all or some plans in a particular order or unordered, or periodically;

- particular conditions are defined to monitor the plan's execution;

- explicit intentions and preferences can be stated for each plan separately.

Basically, an Asbru plan can be seen as a template. This template gets instantiated whenever the plan gets executed. Additionally, more than one instance might be created for a single plan. This pattern can be seen as an analogy to the Class-Object relationship in Object-Oriented Programming.

Since a plan is represented in XML, it is basically readable to humans. But understanding a plan in such a representation needs a lot of training as well as semantic and syntactic knowledge about the representation language. It is cumbersome, and surely not suited for physicians. Therefore, the formal representation needs to be translated into a form familiar to domain experts to be able to communicate the logic of a computerized treatment plan.

\subsection{User Tasks and Scenarios}

To illustrate the different tasks of medical personnel, we created three use scenarios [8] of physicians in protocolbased care.

Scenario 1. Markus Zolte, assistant doctor in training in internal medicine, will be working in the pediatrics department for the next few months and is exploring various treatment methods for new born infants. He informs himself about hyperbilirubinemia by walking through the related treatment protocol. He is interested in the logical workflow and explores the treatment plan. After the first walkthrough of the hyperbilirubinemia protocol, Markus Zolte goes back to the intensive phototherapy part and wants to know in which cases this plan is stopped. He is also interested which part of the complete treatment plan he is viewing right now. Furthermore, he wants to see all other parameters and variables that are getting used in this treatment plan.
Scenario 2. Andrea Habacher, assistent medical director of internal medicine, just completed the treatment of a patient using the controlled ventilation plan. Now, she wants to analyze different parts of the treatment along with measured patient data. She starts by examining how long different phases of the plan took in relation to others. The "handle PCO2 plan" is of particular interest to her. She also wants to see the $\mathrm{PCO} 2$ value for examining relations between plan execution and $\mathrm{PCO} 2$ values. Because there is a significant discontinuity of the $\mathrm{PCO} 2$ value within this plan, she recalls the sub-steps taken in the "handle PCO2 plan". Furthermore, she wants to see when the particular steps were conducted. After that, she is interested in if and how the PCO2 values influenced the "patient-state" parameter.

Scenario 3. Heinrich Kovanic, assistant medical doctor in an intensive care unit (ICU), is currently treating a patient who suffers from hyperbilirubinemia. He examines the "TSB" (total serum bilirubin) and "TSB-change" values and wants to review the patient record for getting basic patient information. After that, he investigates all incoming parameters and encounters a rapid increase of the TSB value that happened two hours ago. He wants to find out which plan or action took place at that time. Furthermore, he examines the parameter constraints defined by the plan conditions. After encountering the reason for the value change, he wants to go back to the current position of plan execution.

Tasks. Summarizing the essentials of these scenarios, three fundamental user tasks can be identified:

- Becoming acquainted with a specific treatment method and observed patient's parameters.

- Guidance in the treatment process (run-time support while treating a patient via monitoring patient status, presenting upcoming treatment steps, and providing a treatment history).

- Analyzing the treatment process (observed data together with treatments).

\subsection{Data Characteristics}

The underlying data for the tasks identified above can be broken down in three categories:

- treatment plan specification data

- treatment plan execution data (instantiation and execution of a treatment plan)

- patient data (time oriented)

Analyzing the type and structure of this data formulated in Asbru yields a number of visualization relevant characteristics: 
- time-oriented data (execution data and planning data including a rich set of time attributes to represent uncertainties)

- logical sequences

- hierarchical decomposition

- flexible execution order (sequential, parallel, unordered, any-order)

- non-uniform element types

- state characteristics of conditions

Starting from this basis of user tasks and data as well as visualization relevant characteristics, we examined related work as highlighted in the upcoming section. Following this, we describe the first step of our user centered development approach, the acquirement of physicians' needs. After that, we bring forward why we chose to introduce a multiple view approach and explain its design in Sections 5-7. Then, we present evaluation and prototype implementation issues. Following that, we describe how users' tasks are supported in Section 9. Finally, we sum up our findings in Section 10 and present work left to be done in future in Section 11.

\section{Related Work}

We investigated related work in the areas of medical treatment planning, information visualization, and commercial medical software as described in the following.

\subsection{Medical Treatment Planning}

Clinical Algorithm Maps. The most widely used visual representation of clinical guidelines are so-called flow-chart algorithms, also known as clinical algorithm maps [15]. A standard for this kind of flow-chart representation has been proposed by the Committee on Standardization of Clinical Algorithms of the Society for Medical Decision Making:

"However, since algorithmic logic is wired implicitly into a protocol, it is difficult to learn an algorithm from a protocol. By contrast, flow-chart algorithms, or clinical algorithm maps, are uniquely suited for explicitly communicating conditional logic and have therefore become the main format for representing a clinical algorithm clearly and succinctly." [34]. The proposed standard includes a small number of different symbols and some rules on how to use them. One additional feature to standard flow-charts are annotations that include further details, i.e. citations to supporting literature, or clarifications for the rationale of decisions.

A big advantage of using flow-charts is that they are well known among physicians and require minimal additional learning effort. A drawback of basic flow-chart representations is their immense space consumption if more complex situations are depicted where overview is lost easily. Temporal information can only be represented implicitly on a very coarse level in terms of an item's relative position within a sequence. Furthermore, flow-charts cannot be used to represent concurrent tasks or the complex conditions as used in Asbru. Clinical algorithm maps were intended to be used on paper and have never been enriched by computer support such as navigation or versatile annotation possibilities.

AsbruView. AsbruView [18, 19] is a graphical tool that supports authoring and manipulation of Asbru plans. AsbruView utilizes metaphors of running tracks and traffic control to communicate important concepts and uses glyphs to depict the complex time annotations used in Asbru. The interface consists basically of two major parts, respectively views - one captures the topology of plans, whereas the second one shows the temporal dimension of plans but no depiction of plan and patient data is possible. The intention of AsbruView is to support plan creation and manipulation. It is not supposed to communicate the combination of logic, structure, and temporal aspects of an Asbru plan and patient data during execution or analysis.

Other Scientific Projects. Other scientific work [35, 5, 28] on visual representations focused on visualizing patient data over time or plan execution over time. Research projects dealing with protocol-based care include GLARE [14], GUIDE [29], Protégé [33], GLIF [25], PROforma [11], and GASTON [9]. (A comprehensive overview of related protocol-based care projects can be found in [26] and [37].)

Only some of the available projects dealing with protocol-based care provide any graphical representations. The listed ones include such graphical representations, but most of them only focus on authoring plans. They use a flowchart- or workflow-like presentation depicting the elements used in their formal representation. A more detailed discussion of the quoted projects can be found in [1].

These tools make authoring clinical protocols easier especially for non-computer-scientists but they use graphical representations which are not familiar to domain experts and mix state and flow-chart characteristics within a single diagram. Understanding such representations and using them for plan authoring requires a considerable amount of learning effort.

Authoring clinical guidelines and communicating complete protocols to domain experts are two rather different tasks with different goals. For guideline authoring, first of all, one can assume a more thorough knowledge of the user in the computer domain. Furthermore, a higher threshold towards acceptable learning effort is likely. In terms of aid for achieving the goal of a completely specified guideline, the user needs an overview of what elements are available for 
constructing it. Additionally, means for data input have to be provided and mechanisms for preventing mistakes in the authoring process should be present. This is in contrast to the goal of communicating the logic of a treatment plan together with temporal aspects and patient state parameters where the presentation of and navigation within guidelines is paramount along with providing easy access to linked information and in-depth explanations.

\subsection{Information Visualization Methods}

Visualizing Logical Sequences. Other possibilities to visualize logical sequences away from flow-charts are Structograms [22], PERT charts, Petri nets, and State Transition Diagrams. These techniques focus on other purposes and some of them are more powerful and expressive than flowcharts. But none of them offers a notion for depicting hierarchical decomposition, flexible execution order, and the state characteristic of conditions together in their basic forms as needed for representing Asbru plans.

Visualizing Hierarchical Data. The most popular techniques for visualizing hierarchical data are Trees. Further techniques for that matter are Treemaps [16] that introduce an additional dimension by proportional space assignment. But these 2D techniques have no notion to depict logical sequences, concurrency, or states.

Visualizing Time-Oriented Data. Time is a very important data characteristic but methods for visualizing time other than in time-series plots are not well known. The probably best known method among them are GANTT charts and their utilized Time Lines. An extension of Time Lines are LifeLines [27, 28] that have been used for example to visualize personal histories. A drawback of these methods is that they mostly work retrospectively, thus only depict temporal attributes in the past. To overcome this limitation, other visualization techniques like Temporal Objects [7], Paint Strips [6], and SOPOs [20, 17] were developed. These techniques can be used to visualize complex notions of time like temporal uncertainties that can be utilized to depict future planning data. The main flaw of the presented techniques is that, except GANTT charts, they cannot depict hierarchies and logical sequences can only be represented implicitly.

\subsection{Commercial Medical Software}

A very high portion of the offered commercial software products in medicine deal with administrative issues such as patient data management or billing. Only very few include any visualization parts and even less offer functionality to aid treatment planning.

We examined a number of non-administrative software products that use graphical representations in general (not only focused on protocol-based care), for the reason of compiling a set of graphical representations most commonly used and that are familiar to most physicians [1].

All of the examined products are rather data-centric and the most popular form of data representation is using tables where numerical and textual data is organized in spreadsheets. None of the investigated products offered a way of visualizing treatment planning logic at all.

We think that besides examining related work on a scientific basis and investigating commercial products it is absolutely necessary to involve end-users from the very beginning. Only this can ensure the incorporation of the users' valuable experience, knowledge, and desires, thus increasing quality and acceptance dramatically. This user-centric development was started by carrying out a user study as described in the following section.

\section{User Study to Acquire Physicians' Needs}

A step of major importance for requirement analysis in our development process was to conduct a user study with eight physicians to gain deeper insights into the medical domain, work practices, application of guidelines in daily work, users' needs, expectations, and imaginations.

Most of the interviewed physicians work at different departments for critically ill patients at the General Hospital of Vienna (AKH Wien). The AKH Wien is a university clinic which means that employed physicians' work also includes scientific research. Conducting an interview took on average about 45 minutes and led to interesting, but not too surprising results and insights. (Detailed results and interview guidelines can be found in [1].)

Fundamental issues for the interviewed physicians were rather practical ones. Most importantly the system has to save time - no one would use a system if it would take more time as working without it. Another major issue is that learning effort for using the system has to be minimal. The system should be intuitive, simple, and clearly structured without complex menu structures or functions.

It became apparent that clinical guidelines are generally depicted by a special form of flow-charts named clinical algorithm maps as proposed in [34] and are widely known. GANTT charts were known among most of our interview partners and half of the interviewed physicians knew LifeLines and PERT charts. LifeLines however, were understood much more easily when asking for the possible meaning of an example.

When summarizing and evaluating the results of our user study, the following fundamental characteristics can be recognized - a simple and transparent structure, intuitive interaction (easy to learn and comprehend), a cleaned up interface, a high level of application safety (undo where possi- 
ble), time saving (allowing quick and effective work), fast, and flexible.

\section{Why Multiple Simultaneous Views?}

As described in Section 1, the underlying data structure we want to communicate to medical domain experts is very complex. Since none of the examined visualization methods can be used to represent all needed data characteristics, we had to decide whether to introduce a new visualization method that allows the depiction of every data aspect in one view or using the approach of multiple views. Multiple views are a well known information visualization technique, whereby a number of representations that focus on different aspects of the data are provided for a common underlying data structure. Fundamental research in formalizing this approach together with its incorporation into the visualization reference model has been carried out by J.C. Roberts [30, 31]. Furthermore, several guidelines for using multiple views in information visualization have been presented by M. Wang Baldonado et al. in [36].

Several reasons led to the clear decision of using multiple simultaneous views. Since we were putting forward a usercentered approach, the goals of providing representations that are easy to comprehend and require as little learning effort as possible were paramount. Therefore, using representations familiar to the domain experts was obvious. Furthermore, we perceived that a single representation would be far too complex, cognitively overwhelming, and surely not optimal to fulfill our prerequisites. Our user study clearly showed that clinical algorithm maps are frequently used in daily work and education of physicians to represent treatment plans. GANTT-charts and LifeLines were identified as quite well known techniques for representing temporal aspects. Since these methods in combination are capable to serve our needs, we chose them as basis for our design.

A further important factor related to this are the different tasks users want to accomplish by using our interactive visualization methods. The three primary tasks of becoming acquainted with a specific treatment method, guidance in the treatment process, and analysis of the treatment process are temporally as well as semantically intertwined, which led to the decision of using multiple simultaneous views rather than sequential ones.

A successful introduction of a multiple view approach in the medical domain has been demonstrated by Zeng and Cimino [38]. They developed a web-based hypermedia system for physicians and clearly showed the advantages of multiple views in the medical domain.

Having introduced the domain prerequisites, data characteristics, user tasks, related work, our user study, and reasons why we chose to use multiple simultaneous views, we now present these views in detail.

\section{Views}

Basically, we divided the underlying data structure along the lines of logical structure and temporal aspects. Hence, we provide a Logical View and a Temporal View along with a QuickView Panel. These distinct views are presented simultaneously and divide the screen in the following manner (see Fig. 1). The QuickView Panel is located on top of the screen displaying the most important patient parameters and plan variables at a prominent position. Below that, the screen is divided vertically by the logical view on the left and the temporal view on the right side. The logical view presents treatment plans in terms of their logical structure (hierarchical decomposition, plan elements, execution order, conditions). The temporal view on the other side focuses on the temporal aspects of treatment plans and monitoring of measured patient data as well as plan variables (temporal aspects of plan elements, temporal uncertainties, hierarchical decomposition). We preferred an integrated approach in contrast to multiple windows due to a more efficient use of screen estate, a less cluttered display, and less necessary user interaction when resizing views.

Table 1 summarizes which data characteristics are visualized by the different views.

\subsection{Logical View}

The logical view on the left part of the screen provides a representation of the treatment plan specification data along with an indication of the current execution position within this plan, which can be considered as run-time attribute of the instantiated treatment plan. The visualization technique is based on the idea of flow-chart-like clinical algorithm maps that are well known amongst physicians. This concept has been extended in order to be able to depict the characteristics of a treatment plan formulated in Asbru.

A set of six visual elements is used to depict the single steps within the body of an Asbru plan - Plan, Userperformed plan, Ask element, Cyclical plan, If-Then-Else Element, and Variable assignment. For depicting plan conditions and the execution order of the plan steps, an enclosing frame was created (see Fig. 2). The topmost bar is filled with the plan color and contains the title of the plan. Below the plan title, the abort condition is shown. It is represented by a red bar having a stop sign icon at the left side. Right besides this icon, the abort condition is printed textually. This condition has the following semantic - if the condition evaluates to TRUE, the current plan gets aborted. Furthermore, this condition is evaluated and checked during the entire execution of all steps in the plan body. The green bar at the bottom of the plan represents the complete condition. It has a checked finish flag icon at its left and contains the complete condition textually. The semantic of 


\begin{tabular}{l|c|c|c}
\hline & Logical View & Temporal View & QuickView Panel \\
\hline Asbru plans & $\bullet$ & $\bullet$ & \\
Time-oriented data & & $\bullet$ & \\
Logical sequences & $\bullet$ & & \\
Hierarchical decomposition & $\bullet$ & $\bullet$ & \\
Non-uniform element types & $\bullet$ & $\circ$ & \\
Conditions & $\bullet$ & & \\
Parameters and variables & & $\bullet$
\end{tabular}

entirely represented $(\bullet)$, partly or implicitely represented ( $\circ$ ), or not represented (empty).

Table 1. Data characteristics in views.

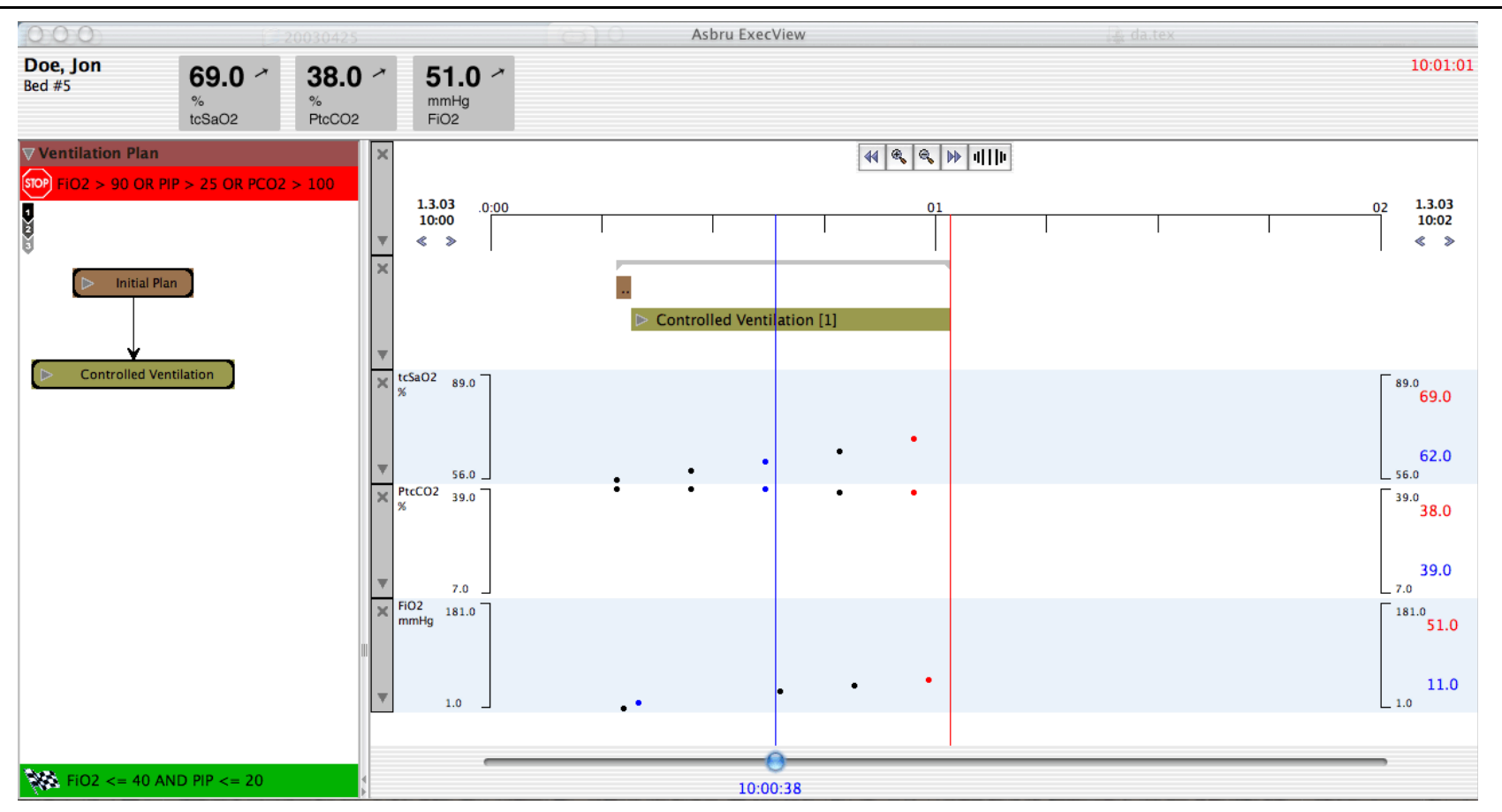

Figure 1. Application window showing the execution of a plan.

this condition is - if and only if this condition evaluates to TRUE, the plan can complete successfully. The largest part of the representation is dedicated to the plan body of the depicted plan along with the execution sequence indicator. Its four possible symbols specify the execution order of the elements within the plan body - sequentially, parallel, anyorder, or unordered.

The visual exploration of a treatment plan is supported by several interactive features. Plan elements that contain sub-elements are indicated by small gray triangles right in front of their labels. By clicking the triangle, the user navigates down the hierarchy, revealing the child elements of the chosen element. This navigational technique is well known from file system viewers as for example the Finder of the Macintosh ${ }^{\mathrm{TM}}$ system.

In order to prevent getting lost within a plan by navigation, two focus +context techniques are applied. Firstly, there is the overview+detail technique that uses a small window containing a downscaled, simplified tree overview where the current position within a plan is highlighted. This 


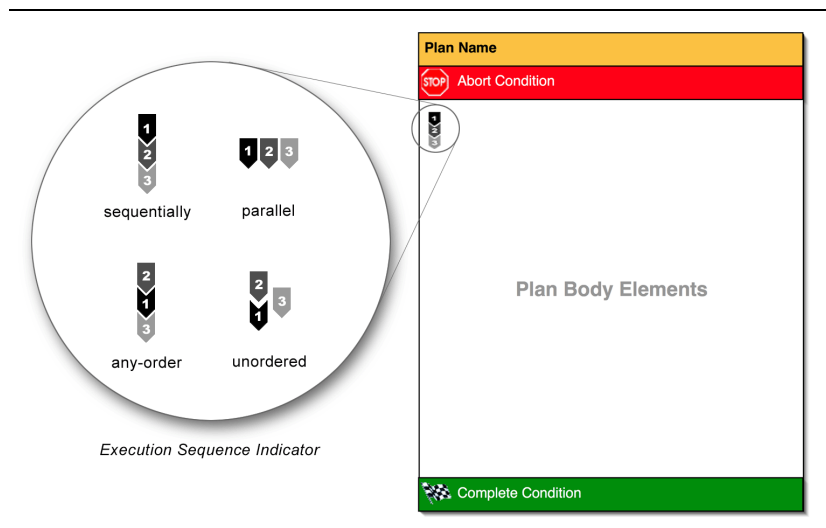

Figure 2. Basic structure and execution sequence symbols.

small overview window can be toggled on or off. The second technique used is the fisheye view which distorts elements that are out of the current focus geometrically by shrinking and moving them (see Fig. 3). This method has been introduced by Schaffer et al. in their work on hierarchically clustered networks [32].

For a comprehensive description of the visualization methods used within the logical view refer to [2].

\subsection{Temporal View}

The temporal representation of treatment plans is based on the idea of LifeLines. This concept has been extended for enabling the display of hierarchical decomposition as well as the complex time annotations used in Asbru. These new visual elements are called LifeLines+ and PlanningLines, respectively. LifeLines+ allow the interactive representation of temporal intervals with hierarchical decomposition and simple element characteristics. On top of that, PlanningLines allow the depiction of temporal uncertainties via a glyph consisting of two encapsulated bars, representing minimum and maximum duration, that are bounded by two caps that represent the start and end intervals (see Fig. 4). Encapsulated bars that can be shifted within the constraints of two mounted caps resemble the glyph's mental model.

The navigation is achieved analogous to the logical view by using small gray triangles which expand and collapse elements. In order to prevent visual overload and an overly cluttered display, expanded elements are shrunk to summary lines and colored in light gray.

The temporal view is used to display the temporal aspects of plans and patient data in the past, present, and future, whereas only plans can be shown in future including temporal uncertainties.

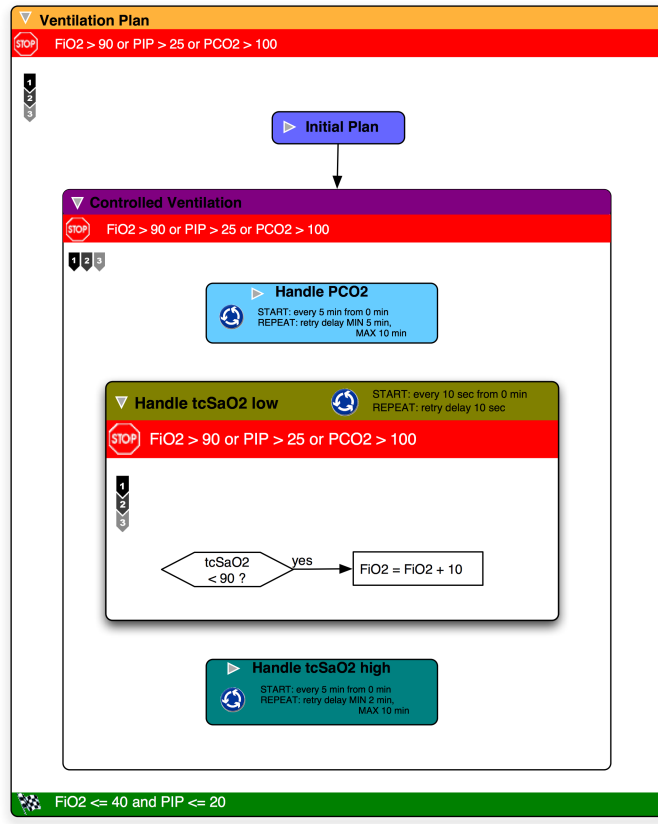

Figure 3. Logical view showing a ventilation plan (fisheye mode).

The temporal view is divided into collapsable facets which can be added and removed dynamically. The most important element of this view is the time scale. It determines the portion of time being displayed. Below that, one facet is displayed containing the temporal aspects of the treatment plan elements followed by several facets containing different plan parameters and variables measured or computed over time. Collapsing facets leads to vertically shrunk and semantically zoomed representations which can be considered as focus +context technique. Another focus+context technique is applied to the time axis itself. Fisheye deformation is used to magnify the focus part of the time scale while the context part is demagnified. This fisheye functionality can be turned on and off via a button above the time scale. Furthermore, the time scale can be zoomed and shifted interactively. Due to the nature of this focus+context technique, where the same data is shown in a different way, the temporal view can also be considered as a multiform view [31].

\subsection{QuickView Panel}

A separate possibility to display currently valid variable and parameter values is the so-called QuickView Panel in the top part of the application window (see Fig. 1). The panel consists of rectangular areas that can be assigned to 


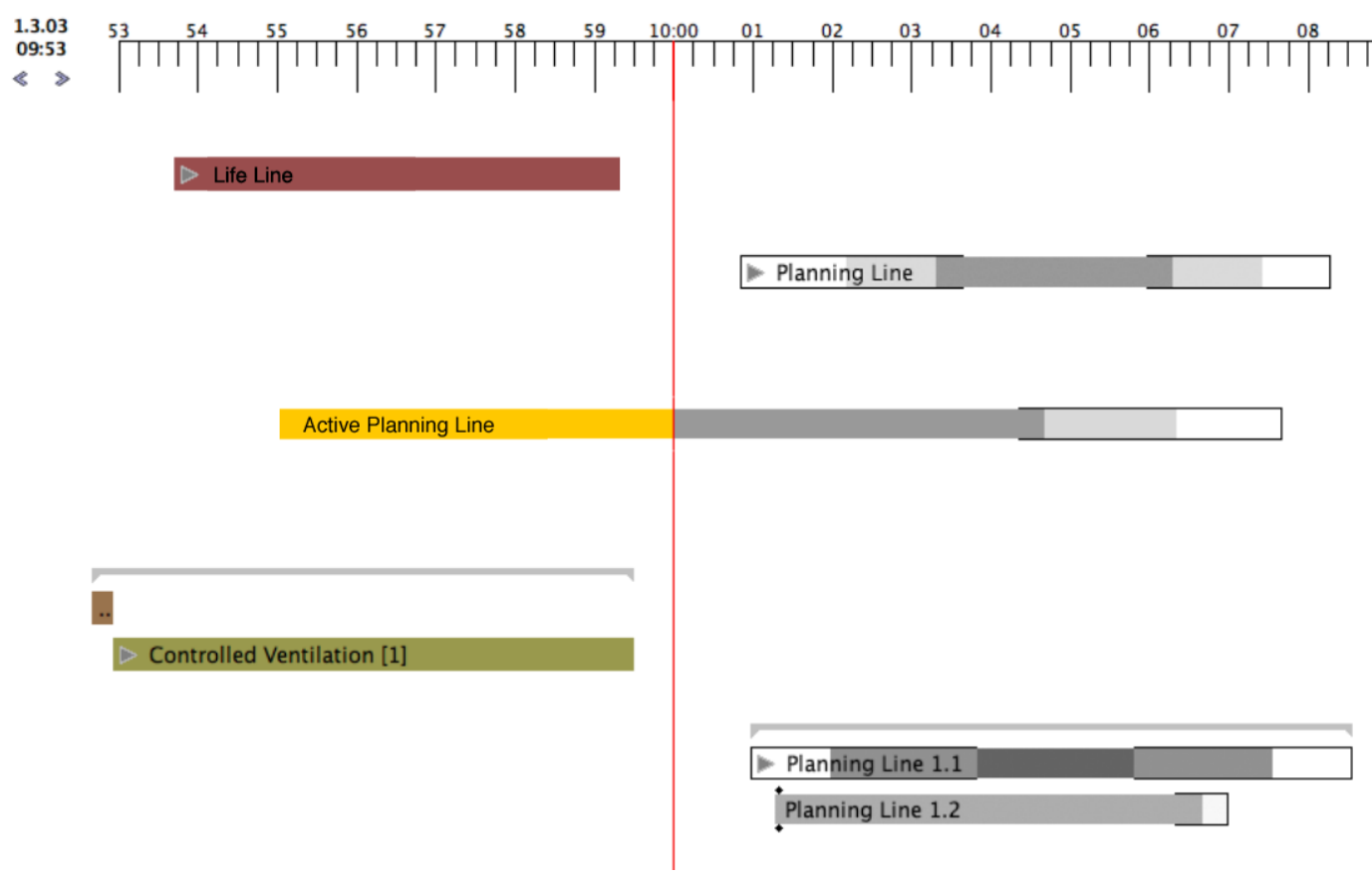

Figure 4. Temporal view elements (LifeLines+, PlanningLines).

the available parameters and variables. A single item shows the current value along with its name, unit, and a trend indicator. Thus, the QuickView Panel allows to monitor the most important values by putting them at a prominent position, enlarged in size and without the need for displaying the complete history in an additional facet.

\section{View coupling}

Logical view and temporal view are tightly coupled in three different ways. Firstly, a common color palette is used among the views for coloring plan elements. This means that plans in the logical view and their instances in the temporal view are colored equally in order to allow for easy recognition of related elements.

The second method of coupling is linking+brushing through synchronous selection. If an element is selected in either the temporal or the logical view, the corresponding element(s) are selected in both views. This ensures a quick recognition and comparison of an element of interest in both views. A special aspect to deal with is the hierarchical decomposition of elements. What to do if an element is selected in one view that is expanded and showing its child elements in the other view? In this case, all child elements belonging to the selected element are selected together with the parent element itself when visible.

This issue leads to the last coupling method of coordinated navigation. In contrast to the already presented meth- ods, navigational procedures within a plan are not propagated to the coupled view, thus providing no automatic synchronization. Instead, view synchronization is user triggered via drag and drop. If the user wants to propagate the current position within a plan from one view to the other, she selects the desired element, moves it to the other view and drops it there. This user interaction initiates a navigation of the selected view to the desired position.

Chris North's snap paradigm [24, 23] uses two basic classes of user interface actions, namely select and navigate. Applied to two views, three distinct combinations are identified for tight coupling: select $\mathrm{A}$ - select $\mathrm{B}$, navigate $\mathrm{A}$ - navigate $\mathrm{B}$, and select $\mathrm{A}$ - navigate $\mathrm{B}$. In our system, linking+brushing is used analog to the select $\mathrm{A}-$ select $\mathrm{B}$ paradigm, but user triggered navigation propagation is not captured by North's system because of its not immediate and not as "tight" characteristic.

Three main reasons led to the decision of using user triggered view synchronization in our case. First of all, it might be confusing if a mouse click in one view is causing major view changes in both views. Secondly, it is often not desired that the current navigation action is propagated. Only distinct navigation allows the comparison of different parts of a treatment plan in the different views. The third reason is avoiding costly computation effort for view transformations especially in case of chained navigational actions, i.e. navigating from the root element to a leaf element. 


\section{View Management}

For coupling the views, a managing entity is needed. This element is embodied by a view manager class that holds references to all views of Asbru plans in the system. These views do not represent plans themselves visually but use other view elements for that matter: LifeLines + and PlanningLines in the temporal view, and the PlanGraph element in the logical view.

A heavily used architectural element for interaction event notification is the Observer pattern [13]. The user interface (UI) event model has the following event types:

- select: A UI element was getting selected.

- expand: A UI element was getting expanded.

- collapse: A UI element was getting collapsed.

- propagate: The propagation of the current selection has been triggered.

These events are encapsulated in a ViewSelectionEvent class and sent to all registered listeners. The event class holds references to the object sending or resending the event as well as to the object originally firing the event. Classes that are interested in receiving such events have to implement a particular listener interface. This interface defines a set of listener methods that are called upon when the associated event types are fired.

Due to the structure of views, a layered dispatch is used for delivering events. This means that events are passed up in the hierarchy as long as they affect the next level. When the root view manager is reached, it passes the event down to all other plan views registered for receiving UI events (see Fig. 5).

\section{Evaluation and Prototype}

The designed methods have been discussed in a review step followed by the implementation of a Java prototype and its evaluation as described in the upcoming sections.

\subsection{Design Review}

When having completed the first "release" version of the conceptual design, we conducted a review session for getting early feedback regarding our design. This early evaluation process was very valuable and reduced the risk of investing time and effort in unfruitful initiatives.

The review was done qualitatively by two experts: one person is a visualization expert having experience in medical software development and the other one is a physician (medical expert) having visualization knowledge.

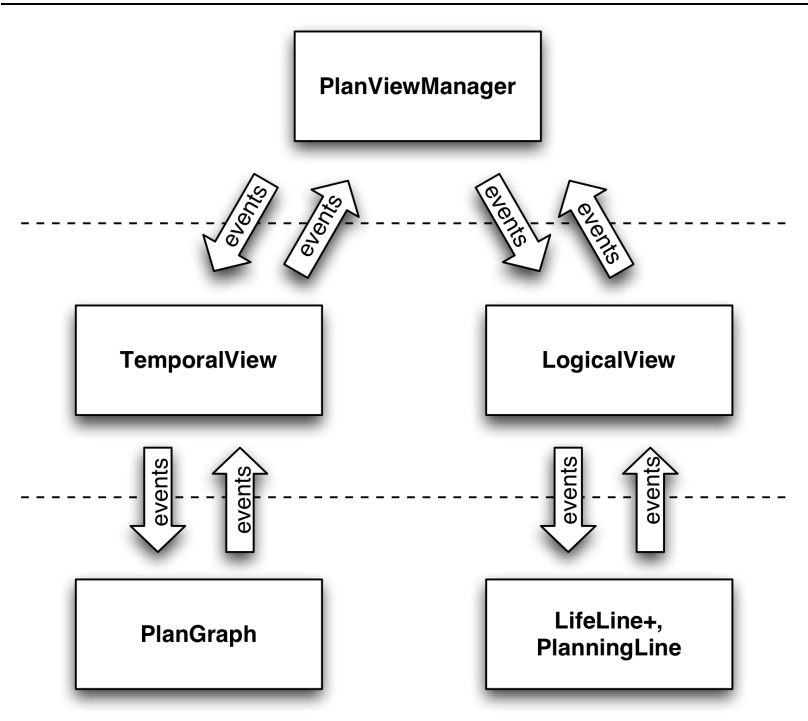

Figure 5. Layered dispatch for views.

The result of the review was very positive, validating our concept, and showing that we were working in the right direction. Only some minor objections were raised about a small number of design issues. The suggestions were incorporated in an improved design (see [1] for detailed results).

\subsection{Prototype Implementation}

As a proof of concept and in order to generate a better impression of interaction issues, we implemented a Java prototype. For depicting the plan step elements in the flow-chart-like part of our representation, we used the graph drawing framework JGraph [3,4]. This is a flexible, small, and powerful package using the Model-ViewController paradigm and is structured analogous to the standard Swing component javax.swing.JTree. All other graphical elements are embedded into the Java Swing standard component framework.

\subsection{Prototype Evaluation}

A scenario-based, qualitative prototype evaluation was carried out by conducting interviews with physicians working in intensive care units. Five of the eight physicians who already participated in the user study at the beginning of this work (see Section 3) took part in the evaluation. The interviews consisted of the four main parts: Introduction, Prototype Presentation, Prototype Testing, and Feedback/Questionnaire [1].

The feedback regarding our design and prototype, given by the interviewed physicians, was very positive. All of them considered the overall structure clear, simple and 
not overloaded. The graphical representations and symbols have been judged to be intuitive and clear, keeping the learning effort relatively low. The interviewed doctors considered the two different views very helpful in working with and exploring treatment plans as well as patient data. Difficulties in relating the views to each other were not perceived.

A particular issue revealed by the prototype evaluation was that the navigation propagation interaction procedure proposed in the original design caused some confusion. Originally, a double-click initiated the navigation propagation which has been replaced by a more intuitive drag and drop interaction.

\section{Supporting Users' Tasks}

So how can Markus Zolte, Andrea Habacher, and Heinrich Kovanic benefit from our visualization methods in accomplishing their work tasks as described in Section 1.2?

Scenario 1. Markus Zolte wants to become acquainted with the hyperbilirubinemia protocol. Therefore, he loads the appropriate Asbru file and maximizes the logical view for examining the logical workflow of the plan. He uses the fisheye view for keeping an overview while exploring different paths of the plan using the small gray triangles for navigation through the hierarchy. When examining the intensive phototherapy part, he deactivates the fisheye view for displaying detail only and reads the abort condition in the read bar on top of the plan to identify cases in which the plan aborts. For getting positional information, he turns on the overview window. Finally, he opens a pull-down menu to see the full list of used parameters and variables.

Scenario 2. Andrea Habacher just completed treatment along the controlled ventilation plan and would now like to analyze the treatment history. She adjusts the zoom factor of the time scale for displaying the complete execution interval and explores the duration and position of the different phases. Furthermore, she uses the small gray triangles at the LifeLines+ to navigate to subplans. For investigating the "handle PCO2 plan", she selects the plan in the logical view and drops it into the temporal view. Subsequently, all instances of the plan are displayed and highlighted. Furthermore, she selects the $\mathrm{PCO} 2$ parameter at a pull-down menu for display in the temporal view. When encountering a significant discontinuity or the PCO2 value in one of the plan instances, she recalls the substeps of the plan by navigating down the hierarchy in the logical view to investigate which substeps of the treatment procedure might have caused this phenomenon. After that, she displays the "patient-state" parameter in the temporal view to examine how the PCO2 value influences it.
Scenario 3. Heinrich Kovanic is currently treating a patient along the hyperbilirubinemia protocol. He displays the "TSB" and "TSB-change" values in the temporal view as well as in the QuickView panel. In order to get basic patient information, he displays the patient record by double clicking the patient's name. After that, he displays all parameters and variables in the temporal view and encounters a rapid increase of the TSB value. He identifies the point in time of this episode by using the time cursor. He selects the plan that has been executed at that time in the temporal view and drops it into the logical view. The logical view navigates to the dropped plan and shows the details of the applied parameter constraints defined by the plan conditions in the upper red and lower green bars. Finally, he double clicks the current time indicator at the upper right of the application window to navigate the temporal view back to the current position of plan execution.

\section{Conclusion}

Our goal was to develop visualization and interaction methods for supporting medical personnel in computerized protocol-based care. To achieve this goal, we had to consider several data aspects like the logic, structure, and temporal constraints of plans as given at design time, data of instantiated plans at execution time as well as patient data in form of parameters and variables. Several reasons led to the decision of introducing multiple simultaneous views for that matter. The trade-offs of multiple views such as the cost of context switching or an increased system complexity are by far outweighed by the benefits gathered. Applying a multiple simultaneous views approach helped to master the complexity of the underlying data structure while using visualization methods well known to the domain experts. We have examined the usefulness of our approach performing a 3-step evaluation process including user study, design reviews, and prototype evaluation.

That visualizing the logic of clinical guidelines is useful to support understanding and exploration of protocols has already been proposed and proved years ago [34, 15]. Clinical algorithm maps are most widely used in medical education and practice for that matter. This form of representation is clear, simple, and easily graspable - thus served as basis in our visualizations for the representation of a plan's logical structure. But it cannot be applied directly to represent Asbru plans because it does not provide a notion for representing hierarchical decomposition, flexible execution order, and state characteristics of conditions. Therefore, we extended this visualization by introducing new element types; an execution sequence indicator, and an enclosing frame containing the plan conditions.

Besides that, visualizing the temporal aspects of already executed plans, currently running plans, and plans to be ex- 
ecuted in future in addition to the logic of treatment plans is vital for analysis and runtime support in medical treatment planning. Key issues of planning are temporal uncertainties inherently related to the temporal dimension. These uncertainties in the form of Asbru's powerful time annotations are visualized in a simple and meaningful way, fully integrated in the LifeLine based representation.

The use of software in contrast to paper allows us to support the process of exploring and understanding treatment plans at a higher level. It enables a meaningful navigation, providing annotations on demand for not overwhelming the viewer, and keeping orientation by using focus+context techniques, thus increasing the flexibility in working with treatment plans. The introduced views focus on different aspects of the data while being tightly coupled to support physicians at their main work tasks.

An additional value, besides communicating plans to domain experts, became apparent during development. The visualization of plans helps to spot problems, bugs, and ambiguities in the formal plan representation which are hard to see and detect otherwise. Furthermore, the visualization serves as an important basis for the communication between medical domain experts and computer scientists.

Moreover, we applied a user-centric approach when developing our visual representation - we involved the endusers from the very beginning by carrying out a user study and evaluated our design as well as our prototype. This increases the quality of design, the user acceptance, and serves as an indicator of the maturity of development. We used a well known graphical representation as basis and introduced a cleaned up interface that has a simple and transparent structure with only a handful of different visual elements which are easy to learn and comprehend. The interaction is carried out intuitively by applying well known techniques from standard software supported by different focus+context techniques for keeping an overview. The most important user requirement of being time-saving is achieved by combining intuitive navigation and rich information presentation in a structured way. This is in contrast to working with paper-based treatment protocols and patient records that are often a mix of text, tables, and graphics, scattered over various pages, making it hard to keep an overview and conceive the logic of a treatment plan.

\section{Future Work}

On a conceptual level, the tradeoff between an integrated approach and using multiple windows should be investigated systematically as well as the pros and cons of user triggered navigation propagation for view coupling. More effort has to be put into actually implementing the full set of introduced design concepts. The most important measure for that matter is to directly extract the visual repre- sentation from Asbru plan files. Furthermore, all proposed focus+context techniques have to be implemented and rich annotation display possibilities should be integrated. A better layout algorithm for plan step elements within the logical view has to be found as well, including smart aggregation of nodes if appropriate. Besides that, the software environment should be enriched by smart lookup of plans available on the system, within a network, or even over the internet.

\section{Acknowledgements}

This project is supported by "Fonds zur Förderung der wissenschaftlichen Forschung - FWF" (Austrian Science Fund), grant P15467-INF.

\section{References}

[1] W. Aigner. Interactive Visualization of Time-Oriented Treatment Plans and Patient Data. Master's thesis, Vienna University of Technology, Institute of Software Technology and Interactive Systems, Vienna, Austria, May 2003.

[2] W. Aigner and S. Miksch. Communicating the Logic of a Treatment Plan Formulated in Asbru to Domain Experts. In K. Kaiser, S. Miksch, and S. Tu, editors, Computer-based Support for Clinical Guidelines and Protocols. Proceedings of the Symposium on Computerized Guidelines and Protocols (CGP 2004), pages 1-15. IOS Press, 2004.

[3] G. Alder. Design and Implementation of the JGraph Swing Component. Technical Report 1.0.6, February 2002.

[4] G. Alder. The Home Page of JGraph, 2002. http://jgraph.sourceforge.net.

[5] C. A. Brandt, S. J. Frawley, S. M. Powsner, R. N. Shiffman, and P. L. Miller. Visualizing the Logic of a Clinical Guideline: A Case Study in Childhood Immunization. Methods of Information in Medicine, 36:179-83, 1997.

[6] L. Chittaro and C. Combi. Visual Definition of Temporal Clinical Abstractions: A User Interface Based on Novel Metaphors. In Proceedings of AIME 01: 8th Conference on Artificial Intelligence in Medicine Europe, Lecture Notes in Computer Science, volume 2101, pages 227-230, 2001.

[7] C. Combi, L. Portoni, and F. Pinciroli. Visualizing temporal clinical data on the www. In W. Horn, Y. Shahar, G. Lindberg, S. Andreassen, and J. Wyatt, editors, Proceedings of the Joint European Conference on Artificial Intelligence in Medicine and Medical Decision Making (AIMDM'99), pages 301-311. Springer, jun 1999.

[8] A. Cooper. The Inmates Are Running The Asylum: Why High Tech Products Drive Us Crazy and How To Restore The Sanity. SAMS Publishing, 1999.

[9] P. A. de Clercq, A. Hasman, J. A. Blom, and H. H. M. Korsten. Design and implementation of a framework to support the development of clinical guidelines. International Journal of Medical Informatics, 64(2-3):285-318, December 2001. 
[10] M. Field and K. Lohr. Guidelines for clinical practice: from development to use. Institute of Medicine, Washington, D.C. National Academy Press, 1992.

[11] J. Fox and R. Thomson. Decision Support and Disease Management: A Logic Engineering Approach. IEEE Transactions on Information Technology in Biomedicine, 2(4):217228, 1998.

[12] P. E. Friedland and Y. Iwasaki. The Concept and Implementaion of Skeletal Plans. Journal of Automated Reasoning, 1(2):161-208, 1985.

[13] E. Gamma, R. Helm, R. Johnson, and J. Vlissides. Design Patterns - Elements of Reusable Object-Oriented Software. Addison-Wesley, Reading, MA, 1994.

[14] A. Guarnero, M. Marzuoli, G. Molino, P. Terenziani, M. Torchio, and K. Vanni. Contextual and Temporal Clinical Guidelines. In Proceedings AMIA Symposium, pages 683-7, 1998.

[15] D. C. Hadorn. Use of Algorithms in Clinical Practice Guideline Development: Methodology Perspectives. AHCPR Pub., 0009(95):93-104, Jan. 1995.

[16] B. Johnson and B. Shneiderman. Treemaps: A Space-Filling Approach to the Visualization of Hierarchical Information Structures. In Proceedings of the IEEE Information Visualization '91, pages 275-282. IEEE, 1991.

[17] R. Kosara, P. Messner, and S. Miksch. Time and Tide Wait for No Diagram. Technical Report Asgaard-TR-2001-2, Institute of Software Technology and Interactive Systems, Vienna University of Technology, Austria, 2001.

[18] R. Kosara and S. Miksch. Metaphors of Movement A User Interface for Manipualting Time-Oriented, Skeletal Plans. Artificial Intelligence in Medicine, 22(2):111-132, 2001.

[19] R. Kosara and S. Miksch. Visualizing Complex Notions of Time. In J. Roberts, editor, Proceedings of the Conference on Medical Informatics (MedInfo 2001), pages 211-215, 2001.

[20] P. Messner. Time Shapes - A Visualization for Temporal Uncertainty in Planning. Master's thesis, Vienna University of Technology, Institute of Software Technology and Interactive Systems, Vienna, Austria, April 2000.

[21] S. Miksch. Plan Management in the Medical Domain. AI Communications, 12(4):209-235, 1999.

[22] I. Nassi and B. Shneiderman. Flowchart Techniques for Structure Programming. SIGPLAN Notices, 8(8):12-26, 1973.

[23] C. North. Multiple Views and Tight Coupling in Visualization: A Language, Taxonomy, and System. In Proceedings CSREA CISST 2001 Workshop on Fundamental Issues in Visualization, pages 626-632, 2001.

[24] C. North and B. Shneiderman. Snap-together visualization: a user interface for coordinating visualizations via relational schemata. In Proceedings of the working conference on Advanced visual interfaces, pages 128-135. ACM Press, 2000.

[25] M. Peleg, A. A. Boxwala, O. Ogunyemi, and et al. GLIF3: The Evolution of a Guideline Representation Format. In Proc. AMIA Annual Symposium, 2000.
[26] M. Peleg, S. Tu, J. Bury, P. Ciccarese, J. Fox, R. Greenes, R. Hall, P. Johnson, N. Jones, A. Kumar, S. Miksch, S. Quaglini, A. Seyfang, E. Shortliffe, and Stefanelli. Comparing Computer-Interpretable Guideline Models: A CaseStudy Approach. The Journal of the American Medical Informatics Association (JAMIA), 10(1):52-68, 2003.

[27] C. Plaisant, B. Milash, A. Rose, S. Widoff, and B. Shneiderman. LifeLines: Visualizing Personal Histories. In Proceedings CHI'96 ACM Conference on Human Factors in Computing Systems, pages 221-227, New York, 1996. ACM Press.

[28] C. Plaisant, R. Mushlin, A. Snyder, J. Li, D. Heller, and B. Shneiderman. LifeLines: Using Visualization to Enhance Navigation and Analysis of Patient Records. In Proceedings of the 1998 American Medical Informatic Association Annual Fall Symposium, pages 76-80, November9-11 1998.

[29] S. Quaglini, M. Stefanelli, G. Lanzola, V. Caporusso, and S. Panzarasa. Flexible guideline-based patient careflow systems. Artificial Intelligence in Medicine, 22(1):65-80, 2001.

[30] J. C. Roberts. On Encouraging Multiple Views for Visualization. In E. Banissi, F. Khosrowshahi, and M. Sarfraz, editors, IV'98 - Proceedings International Conference on Information Visualization, pages 8-14. IEEE Computer Society, July 1998.

[31] J. C. Roberts. Multiple-View and Multiform Visualization. In R. Erbacher, A. Pang, C. Wittenbrink, and J. Roberts, editors, Visual Data Exploration and Analysis VII, Proceedings of SPIE, volume 3960, pages 176-185. IST and SPIE, January 2000.

[32] D. Schaffer, Z. Zuo, S. Greenberg, L. Bartram, J. Dill, S. Dubs, and M. Roseman. Navigating Hierarchically Clustered Networks through Fisheye and Full-Zoom Methods. ACM Transactions on Computer-Human Interaction, 3(2):162-188, 1996.

[33] R. D. Shankar, S. W. Tu, and M. A. Musen. Use of Protégé2000 to Encode Clinical Guidelines. In Proc. AMIA Annual Symposium, 2002

[34] Society for Medical Decision Making. Proposal for Clinical Algorithm Standards. Medical Decision Making, 12(02):149-154, April-June 1992.

[35] E. Tufte and S. M. Powsner. Graphical Summary of Patient Status. The Lancet, 344(8919):386-389, 1994.

[36] M. Q. Wang Baldonado, A. Woodruff, and A. Kuchinsky. Guidelines for using multiple views in information visualization. In Proceedings of the working conference on Advanced visual interfaces, pages 110-119. ACM Press, 2000.

[37] www.openclinical.org. Open Clinical - Knowledge Management for Medical Care, 2003. http://www.openclinical.org.

[38] Q. Zeng and J. J. Cimino. Providing Multiple Views to Meet Physician Information Needs. In Proceedings of the 33rd Hawaii International Conference on System Sciences. IEEE, 2000. 